\title{
Pulsed-High Field/High-Frequency EPR Spectroscopy
}

\author{
Michael Fuhs and Klaus Möbius \\ Institut für Experimentalphysik, Freie Universität Berlin, Arnimallee 14, 14195 \\ Berlin, Germany
}

\begin{abstract}
Pulsed high-field/high-frequency electron paramagnetic resonance (EPR) spectroscopy is used to disentangle many kinds of different effects often obscured in continuous wave (cw) EPR spectra at lower magnetic fields/microwave frequencies. While the high magnetic field increases the resolution of $\mathrm{G}$ tensors and of nuclear Larmor frequencies, the high frequencies allow for higher time resolution for molecular dynamics as well as for transient paramagnetic intermediates studied with time-resolved EPR. Pulsed EPR methods are used for example for relaxation-time studies, and pulsed Electron Nuclear DOuble Resonance (ENDOR) is used to resolve unresolved hyperfine structure hidden in inhomogeneous linewidths. In the present article we introduce the basic concepts and selected applications to structure and mobility studies on electron transfer systems, reaction centers of photosynthesis as well as biomimetic models. The article concludes with an introduction to stochastic EPR which makes use of another concept for investigating resonance systems in order to increase the excitation bandwidth of pulsed EPR. The limited excitation bandwidth of pulses at high frequency is one of the main limitations which, so far, made Fourier transform methods hardly feasible.
\end{abstract}

\section{Introduction}

Electron Paramagnetic Resonance (EPR) spectroscopy has been developed for applications in various fields of physics, chemistry and biology for about 40 years. Until 10 years ago most EPR spectrometers worked at magnetic fields of $0.34 \mathrm{~T}$ and corresponding microwave frequencies of $9.5 \mathrm{GHz}$, measuring the absorption of continuously irradiated microwaves (continuous wave, cw-EPR). Nowadays, the two major trends are the development of advanced pulsed techniques and the use of high magnetic fields (HF-EPR) from $3.4 \mathrm{~T} / 95 \mathrm{GHz}$ up to $12.8 \mathrm{~T} / 360 \mathrm{GHz}$ or even higher. In particular, pulsed EPR at high magnetic fields offers various advantages in investigating for example protein structure and dynamics. However, at high fields the concomitant increase in microwave frequencies imposes strong technical limitations on the possibility of using pulsed techniques. Nevertheless, in the last years the number of pulsed spectrometers at $3.4 \mathrm{~T} / 95 \mathrm{GHz}$ and $5 \mathrm{~T} / 140 \mathrm{GHz}$ has been rapidly increasing and pulsed spectrometers at $7 \mathrm{~T} / 180 \mathrm{GHz}, 10 \mathrm{~T} / 280 \mathrm{GHz}$ and $12.8 \mathrm{~T} / 360 \mathrm{GHz}$ are currently being developed. Generally, there is no hard criterion for defining which magnetic field fulfills the condition for being 'high field'. It depends very much on sample and application. High-field EPR is used for increase in spectral resolution, increase 
in sensitivity for small samples, information on molecular motion at shorter time scales, and the accessibility of large zero-field fine-structure splittings.

EPR is usually applied to systems containing only one or two electron spins. Their resonance lines are often inhomogeneously broadened. The questions and also the reasons for using pulsed instead of cw-EPR are different compared to Nuclear Magnetic Resonance (NMR), although the theoretical treatment is similar. Pulsed high-field/high-frequency EPR is mainly used to measure relaxation times, which gives insight in the dynamics of the spin carrying site and its surroundings. Another goal is to measure hyperfine couplings of nuclei using Electron Nuclear DOuble Resonance (ENDOR) or Electron Spin Echo Envelope Modulation (ESEEM), which gives insight in the structure of the spin environment. Depending on experimental conditions, pulsed methods may provide a sensitivity advantage, sometimes due to underlying theoretical principles, sometimes due to technical reasons. For NMR pulsed techniques are standard by now and are applied to systems containing many nuclear spins which are somehow coupled but nevertheless distinguishable by their chemical shift or gyromagnetic moment. Sophisticated multidimensional pulsed methods often allow, for example, the measurement of selected couplings and distances. Even though in solid-state NMR the spectra of disordered samples might be inhomogeneously broadened by anisotropic interactions, Magic Angle Spinning (MAS) and special pulse sequences allow spectral resolution to a high degree. In NMR it has been demonstrated how advanced pulsed techniques, compared to cw-techniques, can enormously increase information content and sensitivity. In contrast, the thousandfold higher EPR frequencies lead to technical complications which restrict the feasibility of these sophisticated pulse methods.

\section{Interactions measured by EPR}

EPR spectra are sensitive to many different static and dynamic magnetic interactions. Furthermore, most interactions depend on the orientation of the paramagnetic centers with respect to the magnetic field and are, therefore, described by anisotropic interaction tensors. Only for systems which are fast tumbling in solution and thereby exhibit only averaged interactions, or for single crystals, one observes single narrow resonance lines. However, most of the samples are measured in disordered states (frozen solutions or powders) and, therefore, exhibit inhomogeneous line broadening due to overlap of different orientations and anisotropic interactions. Additional inhomogeneous line broadening occurs due to unresolved electron spin - nuclear spin interactions (hyperfine structure). Modulation of magnetic interactions which may be caused by spatial fluctuations, thereby modulating anisotropic magnetic interactions, leads to relaxation effects. The transversal $T_{2}$ relaxation is related to the intrinsic width of a resonance line, the homogeneous line broadening. Often it is a hard task to disentangle all the different effects visible in EPR spectra and to relate them with information on structure and dynamics. 
However, these effects scale differently with magnetic field strength which leads to the strategy of multifrequency EPR and Zeeman magnetoselection. With magnetoselection one selects specific orientations of molecules by resolving their different resonance frequencies due to anisotropic Zeeman interaction even in disordered samples. Furthermore, with the aid of pulsed EPR, it is possible to disentangle homogeneous and inhomogeneous broadening and thereby to gain information on dynamics from the relaxation behavior. Double resonance methods such as ENDOR allow to measure hyperfine interactions even when they are unresolved in inhomogeneously broadened EPR spectra.

One has to distinguish single spin $(S=1 / 2)$, high spin $(S \geq 1)$ and many electron systems (two or more weakly coupled $S=1 / 2$ spins). For $S=1 / 2$ spin systems the most important interactions are the electron Zeeman and the hyperfine interactions. For the other two cases one has to consider also exchange and dipolar coupling. In the following we give a short introduction to the magnetic interactions of $S=1 / 2$ spin systems and refer to standard EPR text books, such as $[1,2]$, for further reading.

\subsection{Electron Zeeman and hyperfine interactions}

The Hamiltonian $\hat{H}$ for the EPR relevant splittings of a $S=1 / 2$ system in a static magnetic vector field $\boldsymbol{B}_{0}$ is given by

$$
\hat{H}=\mu_{B} \boldsymbol{B}_{0} \overline{\overline{\mathrm{G}}} \hat{\boldsymbol{S}}+\sum_{k} \hat{\boldsymbol{S}}\left(\overline{\overline{\mathrm{A}}}_{k}^{\prime}+a_{\mathrm{iso} k}\right) \hat{\boldsymbol{I}}_{k}
$$

where $\mu_{B}$ is the Bohr magneton, $\overline{\overline{\mathrm{G}}}$ is the anisotropic G tensor, $\hat{\boldsymbol{S}}$ the electron spin operator, $\hat{\boldsymbol{I}}_{k}$ the spin operator of nuclear spin $k, \overline{\bar{A}}_{k}^{\prime}$ its anisotropic hyperfine interaction tensor and $a_{\text {isok }}$ is its isotropic Fermi contact interaction. The G tensor contains the $g$ value for the free electron, $g_{e}$, and contributions from the spin-orbit coupling of not completely quenched angular momentum. Because the quenching of angular momentum is related to the electric field the spin carrying molecule is exposed to in its environment, the G tensor is anisotropic and contains information on geometric and electronic structure in an integrated form. Therefore, for quantitative interpretations of measured $\mathrm{G}$ tensors it is often necessary to use complex quantum chemical calculations. Contrary to this, the hyperfine splittings can be directly linked to electronic structure. The isotropic coupling $a_{\text {iso } k}$ is given by $(8 \pi / 3) g \mu_{B} g_{n} \mu_{n}\left|\Psi\left(r_{k}\right)\right|^{2}$ and, therefore, proportional to the probability of finding the electron with wave function $\Psi$ at the position $r_{k}$ of nucleus $k . \overline{\bar{A}}^{\prime}$ contains the dipolar interaction of electron and nuclear spins. By measuring the angular dependence of the interaction energy related to $\overline{\bar{A}}^{\prime}$, which is given by

$$
\frac{\mu_{0}}{4 \pi} \mu_{e} \mu_{n} \frac{3 \cos ^{2} \alpha-1}{r^{3}}
$$

with $\mu_{e}, \mu_{n}$ the magnetic moments of electron and nuclear spins, one can, for instance, determine distance and orientation of hydrogen bonds of quinone anion 


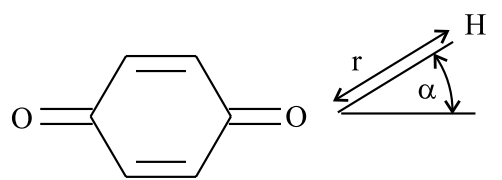

Fig. 1. Hydrogen bond to the oxygen of a quinone anion radical

radicals in various environments (Fig. 1, see Sect. 5.2). This technique can also be successfully applied to proteins with paramagnetic centers such as $\mathrm{Cu}$ atoms, for which complementary NMR studies are blind up to $5 \AA$ around the paramagnetic center.

Diagonalizing (1) by first order perturbation theory and neglecting the nuclear Zeeman term one obtains the following orientation dependent $g$ and $A_{k}^{\prime}$ values

$$
g(\phi, \theta)=\sqrt{\frac{\boldsymbol{B}_{0}}{B_{0}} \overline{\overline{\mathrm{G}}} \overline{\overline{\mathrm{G}}} \frac{\boldsymbol{B}_{0}}{B_{0}}}, \quad A_{k}^{\prime}(\phi, \theta)=\sqrt{\frac{\boldsymbol{B}_{0}}{B_{0}} \overline{\overline{\mathrm{A}}}_{k}^{\prime} \overline{\overline{\mathrm{A}}}_{k}^{\prime} \frac{\boldsymbol{B}_{0}}{B_{0}}} .
$$

For technical reasons in EPR one keeps the frequency of the irradiating microwaves fixed and varies instead the $B_{0}$ value. The resonance fields are given by

$$
B_{\mathrm{res}}=\frac{h \nu}{g(\phi, \theta) \mu_{B}}-\frac{1}{g(\phi, \theta) \mu_{B}} \sum_{k}\left(A_{k}^{\prime}(\phi, \theta)+a_{\mathrm{iso} k}\right) m_{I_{k}} .
$$

Here $m_{I_{k}}$ is the magnetic quantum number of nuclear spin $k$. The five EPR hyperfine transitions of a spin system with $S=1 / 2$ and four equivalent nuclei with $I=1 / 2$ are shown in Figs. $2 \mathrm{a}$ and b. While in EPR one measures the
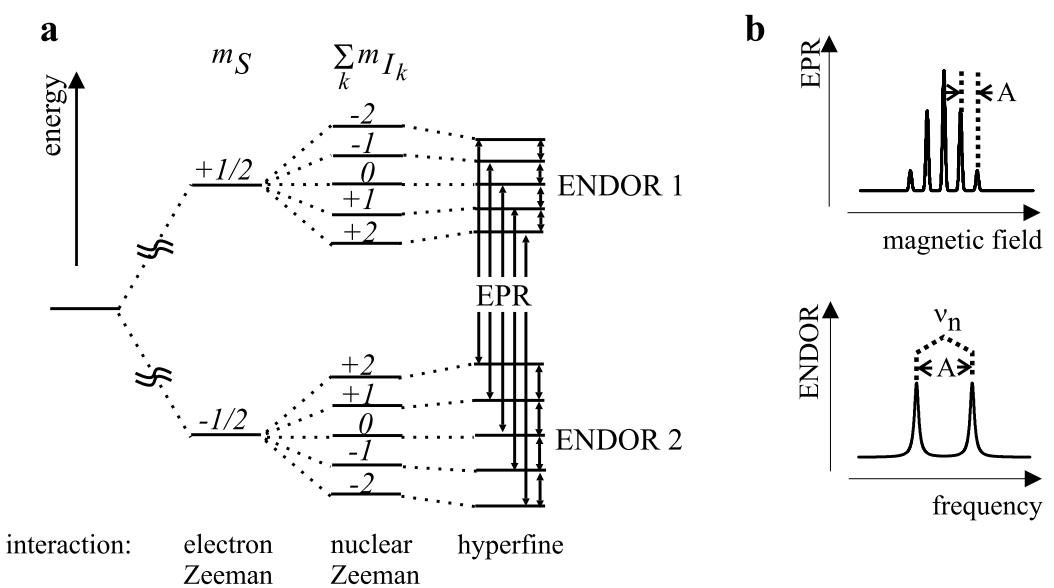

Fig. 2. EPR and ENDOR resonance transitions in a system of one electron with $S=$ $1 / 2$ and four equivalent nuclei with $I=1 / 2 . \Delta m_{S}= \pm 1$ selects the EPR transitions, $\Delta m_{I}= \pm 1$ selects the ENDOR transitions. (a) energy levels. (b) EPR and ENDOR spectra 
electron spin transitions, in ENDOR one monitors the nuclear spin transitions, excited by an additionally applied radiofrequency (rf) field, via its influence on the EPR amplitude. While the number of EPR resonance lines doubles for each additional non-equivalent nucleus, the number of ENDOR lines increases only additively by two. Also for equivalent nuclei, in ENDOR the number of resonance lines is significantly reduced (Fig. 2b). This leads to the resolution advantage of ENDOR for hyperfine structure which in EPR produces overlapping resonance lines.

\subsection{High-field EPR}

As already discussed above, the spectra of disordered samples consist of the overlapping resonance lines of different molecular orientations. Only when the shift in resonance for different molecular orientations is larger than the linewidth of the individual lines, it is resolved in the spectra. When the linewidth contribution due to unresolved hyperfine structure, $\Delta B_{\mathrm{hfi}}$, is dominant resolution is enhanced at higher magnetic fields/microwave frequencies. This is because the hyperfine splitting is independent of $B_{0}$ while the $g$ resolution scales with $B_{0}$. This is shown for a quinone anion radical by spectral simulations at different magnetic fields/microwave frequencies, see Fig. 3a. At $3.4 \mathrm{~T} / 95 \mathrm{GHz}$ the principal values of the $\mathrm{G}$ tensor are well resolved and, by performing additional ENDOR, ESEEM or relaxation time measurement at these resonance fields, one selects only those molecules with their respective principal axis of the $G$ tensor oriented along $\boldsymbol{B}_{0}$. The 'high field' condition to be fulfilled for good magnetoselection is therefore

$$
\frac{\Delta g}{g_{\text {iso }}} B_{0}>\Delta B_{\text {hfi }} \text {. }
$$

At higher fields, resolution is also enhanced for nuclear Larmor frequencies. Therefore, high-field ENDOR is often applied to study systems which contain

$\mathbf{a}$

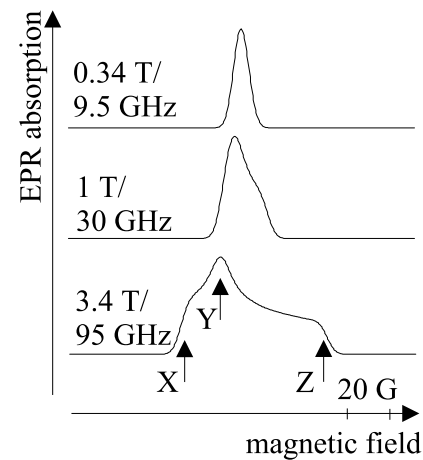

b

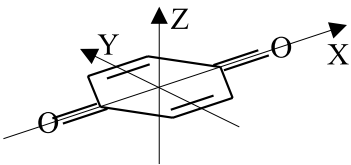

$\mathrm{g}_{\mathrm{XX}}=2.0062$

$\mathrm{g}_{\mathrm{yy}}=2.0051$

$\mathrm{g}_{\mathrm{zZ}}=2.0022$

Fig. 3. EPR on disordered samples of quinone anion radicals. (a) spectral simulations at different magnetic fields/microwave frequencies. The arrows at the bottom spectrum indicate the orientations which dominantly contribute to the EPR absorption at the respective resonance positions. (b) structure of a quinone anion radical with the principal axes of the $\mathrm{G}$ tensor and its principal values 
different sorts of nuclei. At lower fields, the ENDOR spectra, centered at the nuclear Larmor frequency of the respective nucleus, are overlapping while at higher fields they are well separated.

\section{Pulsed EPR}

There are several ways to investigate a spin system by EPR. With continuous wave (cw) EPR one measures the response of continuously irradiated microwaves while sweeping the magnetic field. This is equivalent to the sweep of the microwave frequency at fixed $B_{0}$. On resonance, the microwave energy is partially absorbed. Another possibility is to excite the spin system with a single pulse and to monitor the response. One observes a rotating magnetization and its decay due to dephasing of the individual spins ( $T_{2}$ relaxation) and due to relaxation to thermal equilibrium ( $T_{1}$ relaxation). This is called Free Induction Decay (FID). The Fourier transform of the FID signal is equivalent to the cw-EPR spectrum. Using more than one pulse, because of the non-linearity of spin systems, electron spin echo (ESE) phenomena appear. Pulsed Fourier transform methods offer the advantage of higher sensitivity. Moreover, sophisticated methods using several pulses help to disentangle the various interaction parameters hidden in cw-EPR spectra. This leads to the field of multidimensional magnetic resonance. Particularly interesting is the combination of high magnetic fields/high microwave frequencies with its high $\mathrm{G}$ and high time resolution and pulsed methods. However, the high frequency also leads to limitations of microwave technology and restriction to very basic (field swept) pulsed methods. Some of these field swept methods we introduce in the following. A third method for investigating a spin system by subjecting it to a broadband noise excitation, we will introduce in Sect. 6. For further reading we refer to standard text books [1] for the basic principles and to $[3,4]$ for a thorough treatment of more sophisticated methods.

\subsection{Motion of spins in magnetic fields}

Generally, the motion of spins in magnetic fields is described with the Liouville equation for the density matrix. The observed magnetization $\langle\boldsymbol{M}\rangle$ is given by the expectation value of $-g \mu_{B} \hat{\boldsymbol{S}}$. Restricting oneself to non-coupled spin systems, however, the Bloch equations are a good approximation for many purposes:

$$
\begin{aligned}
& \frac{d M_{x}}{d t}=\frac{g \mu_{B}}{\hbar}(\boldsymbol{B} \times \boldsymbol{M})_{x}-\frac{M_{x}}{T_{2}} \\
& \frac{d M_{y}}{d t}=\frac{g \mu_{B}}{\hbar}(\boldsymbol{B} \times \boldsymbol{M})_{y}-\frac{M_{y}}{T_{2}} \\
& \frac{d M_{z}}{d t}=\frac{g \mu_{B}}{\hbar}(\boldsymbol{B} \times \boldsymbol{M})_{z}+\frac{M_{0}-M_{z}}{T_{1}}
\end{aligned}
$$

The torque of the magnetization in the magnetic field acts on the spin angular momentum which is related to the magnetization by the gyromagnetic factor 
$g \mu_{B}$. The $z$ component of $\boldsymbol{B}$ is the static magnetic field $B_{0}$, the $x$ and $y$ components contain the magnetic component of the circular polarized microwave field with frequency $\omega_{1}$, i.e. $\boldsymbol{B}=\left(B_{1} \cos \omega_{1} t, B_{1} \sin \omega_{1} t, B_{0}\right)$. The Bloch equations may be used to illustrate the different physical meanings of the two relaxation times: The magnetization of the $z$ component decays with the spin-lattice relaxation time $T_{1}$ to its thermal equilibrium value, $\left(0,0, M_{0}\right)$. This process is connected with energy transfer from or to the lattice. The decay of the transversal components is governed by $T_{2}=\left(1 / T_{2}^{\prime}+1 / 2 T_{1}\right)^{-1}$. The processes responsible for $T_{2}^{\prime}$ lead to a dephasing of individual spin packets and do not transfer any energy from the spin states to the lattice.

Without microwave, the magnetization precesses about $\boldsymbol{B}_{\mathbf{0}}$ with the Larmor frequency $\omega_{0}=g \mu_{B} B_{0}$. A microwave field has maximum effect on $\boldsymbol{M}$ when $\omega_{1}$ is equal to the Larmor frequency $\omega_{0}$. In order to treat the effect of the microwave field, it is convenient to work in a coordinate system rotating with the microwave frequency $\omega_{1}$ about $\boldsymbol{B}_{0}$. In this reference frame $\boldsymbol{B}_{1}$ is stationary along the $y_{\text {rot }}$ axis. The magnetization rotates about an effective magnetic field

$$
\boldsymbol{B}_{\mathrm{eff}}=\boldsymbol{y}_{\mathrm{rot}} B_{1}+\boldsymbol{z} \frac{\omega_{0}-\omega}{\omega_{0}} B_{0}
$$

and, therefore, on resonance $\left(\omega_{1}=\omega_{0}\right)$ it rotates about the $y_{\text {rot }}$ axis. A microwave pulse with duration $\tau_{p}$ flips the magnetization of resonant spins with flip angle

$$
\alpha\left(\tau_{p}\right)=g \mu_{B} B_{1} \tau_{p}
$$

\subsection{FIDs, spin echoes and field swept methods}

Following a $\pi / 2$ pulse, the magnetization is rotating in the $x_{\text {rot }} y_{\text {rot }}$ plane with $\omega_{0}-\omega_{1}$. In NMR it thereby induces a current in the receiver rf coils, in EPR the coil is replaced by a microwave resonator. However, due to static inhomogeneities of $\boldsymbol{B}_{\mathbf{0}}$ and stochastic fluctuations of local magnetic fields, individual spin packets have different Larmor frequencies. They dephase, thereby reducing the magnetization.

So far, we have assumed that the microwave pulses have the same effect on all the spins in the sample. This is the prerequisite for performing Fourier transform EPR where a single excitation shot has to contain all spectral information. However, not all spins are on resonance and whether our assumption is true or not depends on the excitation bandwidth of the pulses. Figures 4a and c depict the $x_{\text {rot }}, y_{\text {rot }}$ and $z$ component of the magnetization of an EPR spin ensemble off resonance with $\omega_{0}-\omega_{1}=10 \mathrm{MHz}$, following $10 \mathrm{~ns}$ and $80 \mathrm{~ns}$ pulses, respectively. The pulses have different $B_{1}$, and according to (8) the flip angle is $\pi / 2$. While the 10 ns pulse flips the magnetization almost totally to the $x_{\text {rot }} y_{\text {rot }}$ plane, the $80 \mathrm{~ns}$ pulse flips only a small fraction of the magnetization. Figures $4 \mathrm{~b}$ and $\mathrm{d}$ show the bandwidth of the two pulses. Their FWHM bandwidths are 100 and $12.25 \mathrm{MHz}(3.6$ and $0.5 \mathrm{mT})$, respectively. It is evident that the spins $10 \mathrm{MHz}$ off resonance are not fully excited by the $80 \mathrm{~ns}$ pulse. This is generally the problem in high-field/high-frequency EPR. The $\pi / 2$ pulse lengths e.g. at $95 \mathrm{GHz}$ are 
$\mathbf{a}$

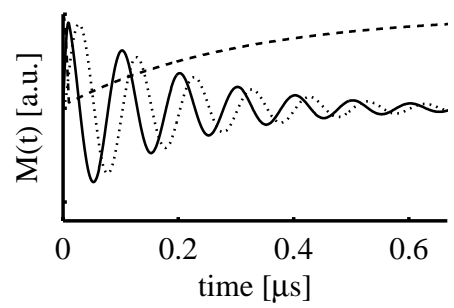

c

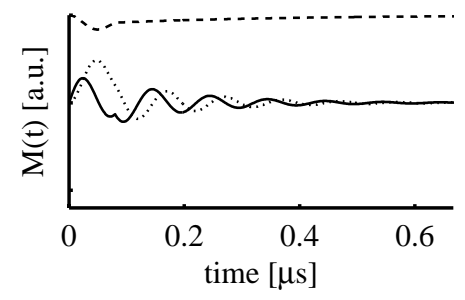

b

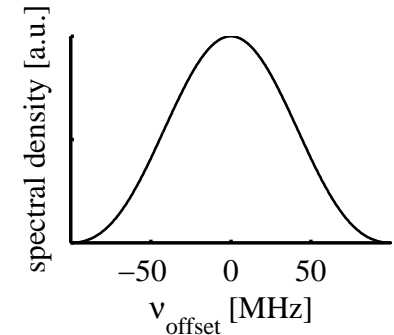

d

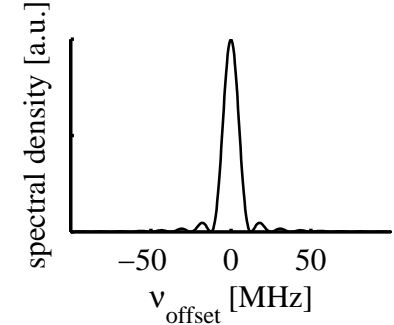

Fig. 4. Magnetization (left) after $\pi / 2$ pulses in the frame rotating with $\omega_{1}$ and pulse excitation bandwidth (spectral density, right). The dashed line is $M_{z}$, the solid line $M_{x_{\text {rot }}}$, the dotted line $M_{y_{\text {rot }}}$. The spins are $10 \mathrm{MHz}$ off resonance, $T_{1}=T_{2}^{\prime}=0.3 \mu \mathrm{s}$. $(\mathbf{a}, \mathbf{b}) \tau=10 \mathrm{~ns}, B_{1}=0.89 \mathrm{mT}$. (c, d) $\tau=80 \mathrm{~ns}, B_{1}=0.11 \mathrm{mT}$. The distortions in $\mathbf{c}$ during the first $80 \mathrm{~ns}$ are caused by the long microwave pulse

generally longer than $40 \mathrm{~ns}$, often about $80 \mathrm{~ns}$ and even longer ${ }^{1}$. EPR spectra of typical organic radicals with resolved $\mathrm{G}$ tensors are often broader than 5 or $10 \mathrm{mT}(140$ or $280 \mathrm{MHz})$. Therefore, Fourier transform EPR is not possible for these samples. Nevertheless, pulsed methods allow to burn holes in the resonance lines and, by sweeping $B_{0}$, one can scan step by step the response to the pulses for all spins in the sample. Some of the field swept pulsed methods are shown in Fig. 5.

A second pulse with flip angle $\pi$, following the first $\pi / 2$ pulse at time $\tau$, refocuses the magnetization to the electron spin echo (ESE) at time $2 \tau$ (Fig. 5a). In field swept methods the echo intensity is integrated for each magnetic field step. The integrated echo intensity over the magnetic field is basically equivalent to the absorption cw-EPR spectrum. Compared to cw-EPR spectra, ESE detected EPR spectra are insensitive to phase distortions and saturation effects. However, only the spins subject to the same static perturbations before and after the $\pi$ pulse refocus, while stochastic perturbations lead to an echo decay with relaxation time $T_{2}$. Therefore, using different $\tau$ it is possible to probe $T_{2}$ (Fig. $5 \mathrm{~b}$ ). The lineshapes of ESE detected spectra depends on $\tau$ when $T_{2}$ varies over the spectrum (see Sect. 5.1). Additionally, the echo decay might be modulated due

\footnotetext{
${ }^{1}$ Freed and coworkers at Cornell have started to use high power extended interaction oscillators (EIO) at $95 \mathrm{GHz}$ which allow them to use much shorter pulse lengths. However, the costs of EIOs at such high frequencies are very high (private communication). Before EIOs have been also used at $140 \mathrm{GHz}$ [5]
} 

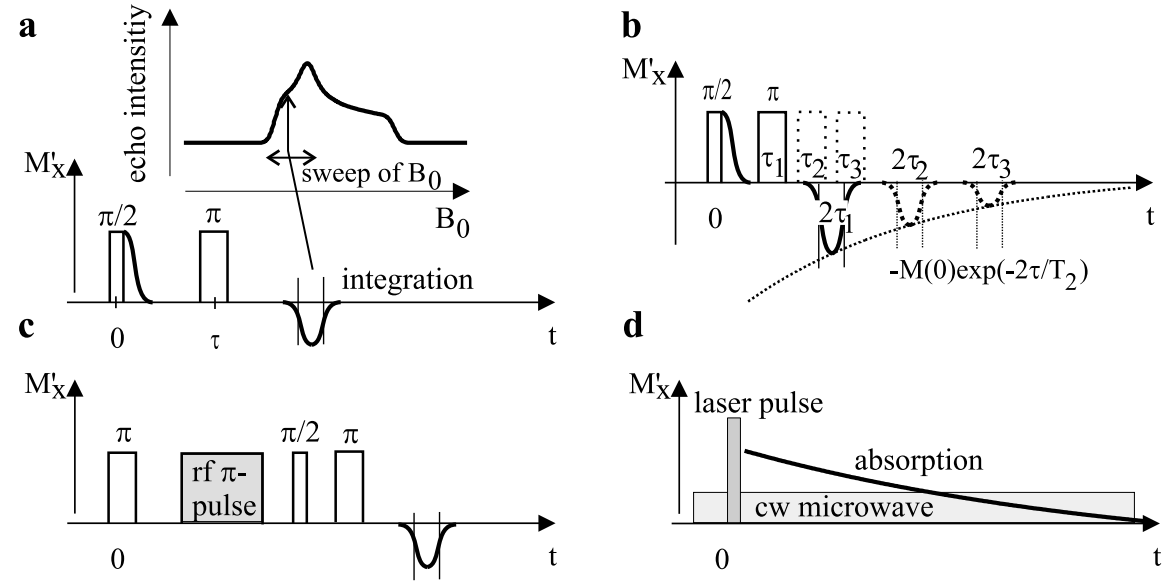

Fig. 5. Frequently used methods for pulsed high-field EPR. The measured magnetization is shown by bold line. The relevant part is integrated as indicated by the two vertical lines. (a) Electron-Spin-Echo (ESE) detected EPR. The integrated echo intensity is monitored over a magnetic field sweep. (b) Measurement of two-pulse echo decay. The echo intensity decays with relaxation time $T_{2}$ and might be modulated due to hyperfine structure (ESEEM). (c) Davies ENDOR. (d) Time-resolved EPR (TREPR)

to hyperfine structure. This leads to Electron Spin Echo Envelope Modulation (ESEEM). ENDOR can be measured in cw as well as in pulsed mode. Because pulsed ENDOR has some technical advantages, it is mainly used in high-field EPR. Figure $5 \mathrm{c}$ shows the Davies ENDOR pulse sequence. The first $\pi$ pulse inverts the electron spin magnetization. This inversion is partially destroyed when the second rf pulse is resonant with a nuclear spin transition. The following spin echo sequence probes the resulting magnetization, which is monitored during the sweep of the frequency of the rf pulse. Time-resolved EPR (TREPR) shown in Figure 5d is not a pulsed method, but it needs, nevertheless, very high time resolution less than $50 \mathrm{~ns}$. This is normally also provided by pulsed EPR spectrometers. In this experiment one probes the absorption of cw-microwave by transient paramagnetic intermediates generated after photo excitation. If relaxation times are slow enough, the transient states can be probed also by a spin echo sequence.

\section{Instrumentation}

The basic setup of an EPR spectrometer is straight forward. One needs a magnet, a transmitter, which provides the microwave field, pulsed and $\mathrm{cw}$, a microwave resonator and a receiver, which has to have the required time resolution. The optimum setup depends on the desired application and purpose. In recent years, a lot of different high-field/high-frequency spectrometers have been developed, pulsed as well as cw. The spectrometers vary in frequency, in time resolution, 
in sensitivity and in microwave output power, which determines the $\pi / 2$ pulse length. A good overview on pulsed high-field EPR instrumentation and sensitivity consideration is given in $[6,7]$.

The sensitivity depends mainly on the resonator design and construction and on the noise figure of the detector. The most sensitive setups use cylindrical cavities as resonators and heterodyne detection. They use microwave mixers which convert the high-frequency signal to an intermediate frequency between 0.5 and $10 \mathrm{GHz}$. Sensitivity is limited by the noise figure of the microwave mixer (typically $9 \mathrm{~dB}$ at $95 \mathrm{GHz}$ ), the amplitude and phase noise of the microwave source which is transmitted to the detector via the local and signal arms of the microwave bridge. Furthermore, sensitivity scales linearly with the quality factor $Q$ of the resonator (typically $Q=2000$ at $95 \mathrm{GHz}$ for cylindrical cavities), and quadratically with the filling and conversion factors. For pulsed EPR, the conversion factor $c$ becomes more important than the filling factor, because it gives the ratio of incident microwave power to the magnetic field strength of the microwave at the center of the resonator, $c=B_{1} / \sqrt{Q P_{\mathrm{mw}}}$. Therefore, the higher the conversion factor, the shorter the pulse lengths $\tau_{p}$ (see (8)) for the available microwave power. This is crucial for samples with short $T_{2}$ relaxation times. In an ESE experiment (Fig. 5a) the delay time $\tau$ between the two pulses cannot be too short because otherwise the echo at $2 \tau$ overlaps with the second pulse or is buried in the spectrometer dead time after the second pulse (typically $10-50 \mathrm{~ns}$ at $95 \mathrm{GHz}$ ). On the other hand, for high sensitivity of pulsed EPR $2 \tau$ should be shorter than $T_{2}$. For this reason it is very important to provide high microwave power output. At $95 \mathrm{GHz}$, typical output powers are between $4-200 \mathrm{~mW}$. The shortest $\pi / 2$ pulse lengths are about 40 ns. As mentioned above, the excitation bandwidth of these pulses is not sufficient for most samples and, consequently, it is often not possible to perform Fourier transform high-field EPR.

On the one hand, when going to higher fields/frequencies the filling and conversion factors increase leading to higher sensitivity for small samples such as single crystals. However, there are many technical problems related with high microwave frequencies, for instance output power and noise figures of the microwave components, and the theoretical sensitivity increase can hardly be realized. Furthermore, the losses in standard rectangular waveguides become enormous and one has to use quasioptical setups instead. A cylindrical cavity at $95 \mathrm{GHz}$ has a diameter of only $3 \mathrm{~mm}$. The sample diameter is about $0.2 \mathrm{~mm}$ for aqueous and $0.8 \mathrm{~mm}$ for samples with low dielectric absorption. These values scale with wavelength, and at very high frequencies one has to use Fabry-Perot resonators instead of single-mode cavities, or even no resonators at all. In such cases the conversion factor drastically reduces and pulsed experiments become much more difficult to perform. The mechanical complications, related to the short wavelength, induce further noise by vibrations, particularly of the microwave coupling. This is a very sensitive component because it matches resonator impedance to the waveguide impedance. It has to be variable because resonator impedance may change with sample and temperature. 
Pulsed ENDOR setups need additional rf coils in order to apply the $\mathrm{rf} \pi$ pulse. The Larmor frequency of protons in $3.4 \mathrm{~T} / 95 \mathrm{GHz}$ spectrometers is $140 \mathrm{MHz}$. The main problems are penetration depth through the resonator walls which, therefore, have to be slotted, matching of the rf circuit in order to allow high power pulses over a bandwidth of at least $20 \mathrm{MHz}$, mechanical and heating problems. The latter two points make pulsed ENDOR at high fields more feasible than cw-ENDOR.

\section{Applications to photosynthetic reaction centers and model systems}

Photosynthetic reaction centers in cell membranes consist of a protein backbone and embedded cofactors. During the primary steps of photosynthesis after photo excitation of chlorophyll electron donors $(\mathrm{P})$, an electron is transfered over a chain of cofactors which leads to charge separated states and, thereby, to trans-membrane potentials. Thus, light energy is converted with high quantum yield, first to electric and afterwards to chemical energy. EPR has been used since long in the investigation of structure and dynamics of photosynthetic reaction centers of bacteria and green plants. It was, for example, applied to the doublet radical of the primary electron donor $\mathrm{P}^{+} \bullet$ of the bacterial reaction center of Rhodobacter sphaeroides, to doublet radicals of the two quinone acceptors, $\mathrm{Q}_{\mathrm{A}}^{-\bullet}, Q_{\mathrm{B}}^{-\bullet}$, and to the intermediate radical pair state during electron transfer, $\mathrm{P}^{+} \bullet \mathrm{Q}_{\mathrm{A}}^{-} \bullet$. Of special interest is the geometric structure, i.e. the orientation and distance of the cofactors with respect to each other and to the membrane, their dynamics in their respective binding pocket, and its relation to electron transfer characteristics. For an exhaustive overview of EPR work related to photosynthetic reaction centers we refer to [8]. In the following we want to illustrate the application of relaxation time and ENDOR measurements to determine structure and dynamics of $\mathrm{Q}_{\mathrm{A}}^{-\bullet}$ in the binding pocket of bacterial reaction centers, as well as the application of time-resolved EPR to structural studies of model systems for photosynthetic electron transfer.

\subsection{Molecular motion studied by relaxation-time measurements}

The 2D spectrum in Fig. 6a shows the echo decay, measured by the pulse sequence in Fig. 5b, with different pulse separation times $\tau$ and magnetic field values [9]. Because the echo decay has different time constants at different magnetic field positions, 1D spectra at different pulse spacing times $\tau$ have different line shapes (Fig. 6b, compare solid and dotted lines). Because at $3.4 \mathrm{~T}$ the highfield condition (5) is fulfilled for $\mathrm{Q}^{-\bullet}$, the different echo decays can be assigned to specific molecular orientations. In the figure the resonance positions for the orientations along the principal axes of the $\mathrm{G}$ tensor are indicated. Magnetoselection works well for the $g_{x x}$ and $g_{z z}$ orientation while intermediate orientations overlap at the $g_{y y}$ field position. The echo decays are mono exponential, with 
the transversal relaxation time $T_{2}$ as time constant (see Sect. 3). The $T_{2}$ values extracted from the 2D spectrum are shown in Fig. 6c.

The transversal relaxation ( $T_{2}$ processes) is caused by stochastic modulation of resonance frequencies. Two quantities characterize the relaxation: (i) the time scale of the stochastic process, and (ii) the depth of the frequency modulation. Under certain conditions, a stochastic process is described with a correlation function $G(\tau)$, which is the probability of finding the system at time $t=\tau$ in the same state as at time $t=0$. For Markov processes, $G(\tau)$ decays exponentially with the correlation time constant $\tau_{r}$ which is a measure for the time scale of the modulation. The mathematical treatment of relaxation processes for the case of fast motion, i.e. $\tau_{r}^{-1} \gg$ modulation depth in frequency units, was described by Redfield [10] and covered in most EPR books (see e.g. [1]). For slower motions the treatment becomes more difficult. The treatment of anisotropic small-angle fluctuations and its relation to ESE-detected EPR spectra was discussed in [11$14]$.

It is assumed that the $\mathrm{Q}_{\mathrm{A}}$ is subject to small-angle fluctuations in its binding pocket. The depth of the modulation of the resonance frequency depends, therefore, on the amplitude of the fluctuations and on the functional relationship of fluctuation angle and resonance frequency.

In the case of $\mathrm{Q}_{\mathrm{A}}^{-\bullet}$, the modulation in resonance frequency is caused by the dependence of the effective $g$ value on molecular orientation. The relaxation is described in the fast-motion regime [9] and $T_{2}$ is related to the rms average
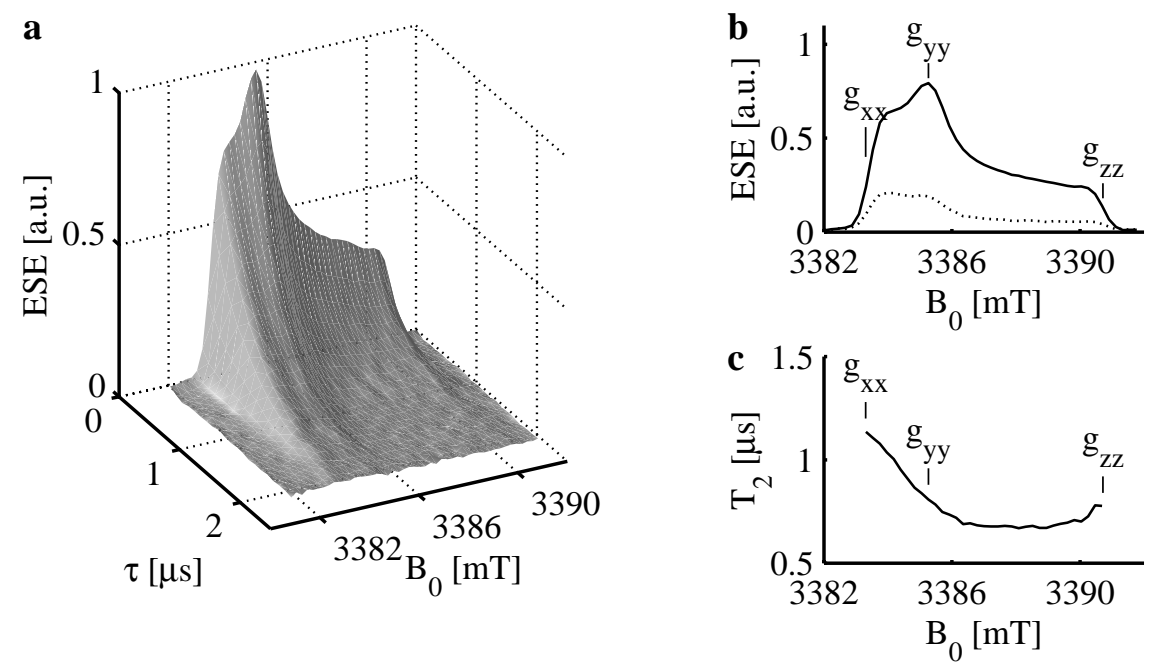

Fig. 6. Study of molecular motion of $\mathrm{Q}_{\mathrm{A}}^{-\bullet}$ in its biding pocket of the photosynthetic reaction center of Rhodobacter sphaeroides at $120 \mathrm{~K}$ with ESE detected EPR [9]. a: 2D spectrum versus $B_{0}$ and $\tau$ (see Fig. 5b). b: $1 \mathrm{D}$ spectrum for $\tau=300 \mathrm{~ns}$ (solid) and $\tau=900 \mathrm{~ns}$ (dotted). c: $T_{2}$ relaxation time versus $B_{0}$ as extracted from $\mathbf{a}$. 
fluctuation amplitude, $\left\langle\delta g^{2}\right\rangle$, by

$$
1 / T_{2}=\mu_{B}^{2} \tau_{c} B_{0}^{2}\left\langle\delta g^{2}\right\rangle .
$$

Because of magnetoselection the dependence of $T_{2}$ on resonance position reveals fluctuation amplitudes for different molecular orientations. Because of the quadratic dependence of $1 / T_{2}$ on $B_{0}$, the resolution for motional anisotropies is increased at higher fields. One can simulate spectra such as in Fig. 6c and thereby relate them with motional anisotropy of the $\mathrm{Q}_{\mathrm{A}}$ acceptor and with characteristics of the binding pocket in the protein. It was shown that, because at $120 \mathrm{~K} T_{2}$ is largest at the $g_{x x}$ field position, $\mathrm{Q}_{\mathrm{A}}$ is fluctuating around the $x$ axis [9]. Of special interest are motional anisotropies due to H-bonding [9], the comparison of different models for anisotropic motion and the comparison of the motional anisotropies of the two different quinone acceptors, $\mathrm{Q}_{\mathrm{A}}^{-\bullet}$ and $\mathrm{Q}_{\mathrm{B}}^{-\bullet}[15]$. Furthermore, studying the temperature dependence reveals changes of motional modes and changes in H-bond lengths [15].

\subsection{Hydrogen bonding studied by ENDOR}

The three ENDOR spectra of $\mathrm{Q}_{\mathrm{A}}^{-\bullet}$ shown in Fig. 7 have been measured using the Davies ENDOR pulse scheme from Fig. 5c. Three different EPR resonance positions have been selected corresponding to the molecular orientations of the principal axes of the $\mathrm{G}$ tensor [16]. By means of this magnetoselection, it is possible to determine the isotropic values and the principal values of hyperfine tensors and their orientation with respect to the $\mathrm{G}$ tensor. By comparing the results with ENDOR on quinone radicals in different environments [16] and measurements at different magnetic fields/frequencies, it was possible to assign

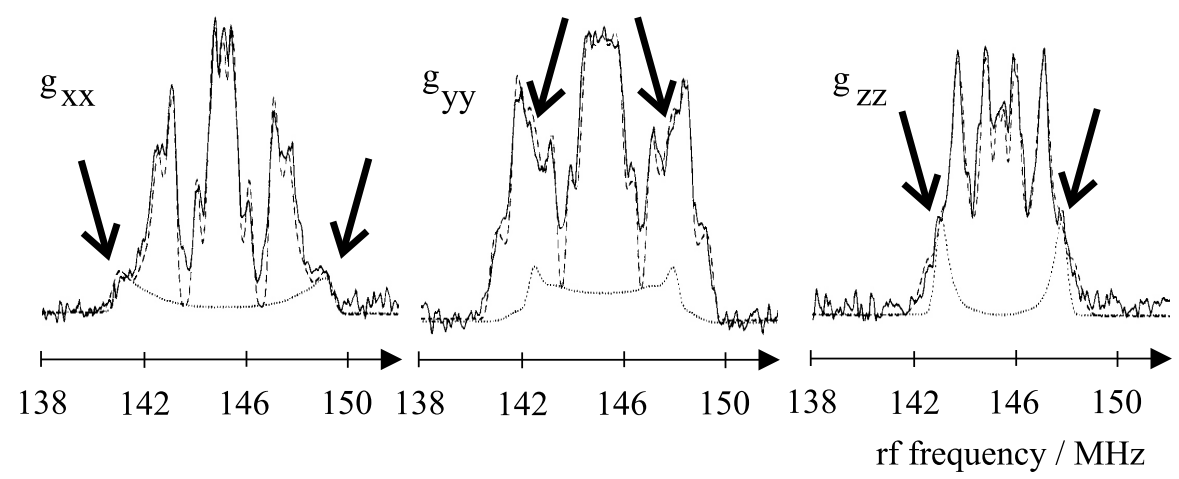

Fig. 7. ENDOR spectra of quinone anion radical $Q_{\mathrm{A}}^{-\bullet}$ in the binding pocket of the photosynthetic reaction center of Rhodobacter sphaeroides. The picture is adapted from [16]. The ENDOR spectra are measured at the three different EPR resonance fields indicated by the arrows in Fig. 3 (bottom). From spectra simulations (dotted lines) the hyperfine couplings (indicated by arrows) could be deduced. They are related to hydrogen bonds of the quinone to amino acid residues of its binding pocket 
the ENDOR lines indicated by arrows in Fig. 7 to a strong H-bonded proton. Using (2), it was possible to suggest the partners involved in the H-bond as well as their distance.

\subsection{Electron transfer and spin dynamics studied by TREPR}

Time-resolved EPR (TREPR) is used to observe states immediately after their creation in chemical reactions or after photo excitation (see $[18,19]$ ). Generally, these states are not in thermal equilibrium but spin polarized, i.e. the population of the states does not reflect Boltzmann distribution. The EPR intensity depends on both transition probability and population difference. Therefore, resonance intensities on spin polarized systems may be drastically enhanced and appear in absorption as well as in emission.

TREPR was successfully applied to study paramagnetic intermediates of the photosynthetic photo cycle, such as the $\mathrm{P}^{+} \bullet \mathrm{Q}_{\mathrm{A}}^{-\bullet}$ state. In contrast to freely diffusing radicals in solution, the two radicals of this radical pair have fixed distance and orientation. The dipolar and exchange coupling are small compared to the differences in local magnetic fields ( $G$ tensors), and the radicals are, therefore, weakly coupled. One observes the resonance lines of the individual radicals split by the dipolar coupling. In this case, the spectra can be well described using a static correlated coupled radical pair (CCRP) model [20]. The higher the magnetic field and microwave frequency, the more the spectrum is determined by the anisotropic $\mathrm{G}$ tensors and the higher is the accuracy with which distance and orientation of the radical-pair partners can be determined.

In Fig. 8 TREPR experiments on the radical pairs of biomimetic model systems for photosynthetic electron transfer are shown [17]. The system consists of a porphyrin electron donor which, after photo excitation, transfers one electron to the quinone acceptor covalently linked to the porphyrin by a cyclohexylene bridge. After the porphyrin is photo-excited to its singlet state, inter-systemcrossing populates the triplet state from which electron transfer takes place, i.e.

$$
\mathrm{PQ} \rightarrow \mathrm{P}^{S 1} \mathrm{Q} \rightarrow \mathrm{P}^{T} \mathrm{Q} \rightarrow \mathrm{P}^{+\bullet} \mathrm{Q}^{-\bullet} \rightarrow \mathrm{PQ}
$$

By varying donors and acceptors and investigating longer chain systems (for the porphyrin-quinone type systems up to two porphyrins and two quinones) one can study spin dynamics and the conditions for highly efficient electron transfer [21-23]. The spectra have been obtained by measuring the absorption of cwmicrowave after the laser pulse (see Fig. 5d). It is also possible to detect the transient magnetization using ESE detected EPR, sometimes even with higher sensitivity [24].

The spectra of the dyad system in Fig. $8 \mathrm{~b}$ at $0.34 \mathrm{~T}$ could be qualitatively simulated using the CCRP approach (Fig. 8c). However, the simulation of the TREPR spectrum at $3.4 \mathrm{~T}$ shows large differences to the experimental spectrum. From this it could be shown that, in contrast to the radical-pair in the natural photosystem, the radical-pair partners in the model system are strongly coupled to a triplet state. Moreover, the spectral characteristics are due to spin lattice 


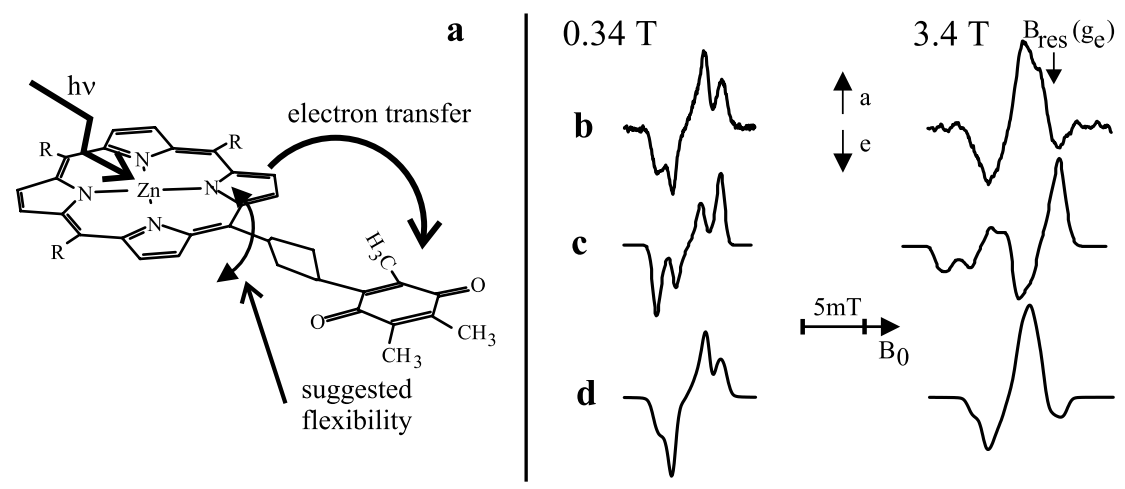

Fig. 8. TREPR on radical pairs $\mathrm{P}^{+\bullet} \mathrm{Q}^{-\bullet}$ of a model system for photosynthetic electron transfer [17]. (a) structure of the porphyrin (P)- cyclohexylene bridge- quinone (Q) system. The molecular flexibility indicated was suggested from the TREPR results. (b) TREPR spectra in frozen solution (ethanol/toluene) $2 \mu \mathrm{s}$ after the laser pulse. (c) simulations as weakly coupled correlated radical pair (small exchange interaction $J_{m}$ athrmex). (d) simulation as strongly coupled radical pair with orientation dependent $T_{1}$ relaxation

$\left(T_{1}\right)$ relaxation effects combined with fast electron recombination [17]. The relaxation effects are associated to anisotropic small-angle fluctuations and intra molecular flexibility. This model led to the simulations shown in Fig. 8d. It could be concluded that the life time of the charge separated state, i.e. the quantity which has to be optimized for artificial photosynthesis with high quantum yield, is limited by these small-angle fluctuations and concomitant relaxation effects.

\section{$6 \quad$ Fourier-transform EPR using broad-band stochastic excitation}

One of the main problems of high-field EPR is the lack of sufficient microwave power. Therefore, the excitation bandwidth of the pulses is often too small for conventional Fourier-transform EPR (see Sect. 3.2). We now present an alternative method for investigating linear and non-linear responses of spin systems. The bandwidth of microwave irradiation is increased by using broad-band noise sources. Noise can be generated by fast stochastic or pseudostochastic phase switching of the microwave irradiation. The stochastic resonance method was introduced to NMR in 1970 by Ernst [25] and Kaiser [26] who have shown that the sensitivity is the same as that of pulsed magnetic resonance, while the required power is reduced by the ratio of pulse length and spin lattice relaxation time. The spectral information is obtained by cross-correlating the excitation with the response of the spin system. This is theoretically equivalent to dispersive infrared spectroscopy in Michelson type interferometers.

Figure 9a shows the first $200 \mathrm{~ns}$ of a pseudostochastic phase switching sequence used for stochastic high-field/high-frequency EPR [27]. The FWHM spec- 

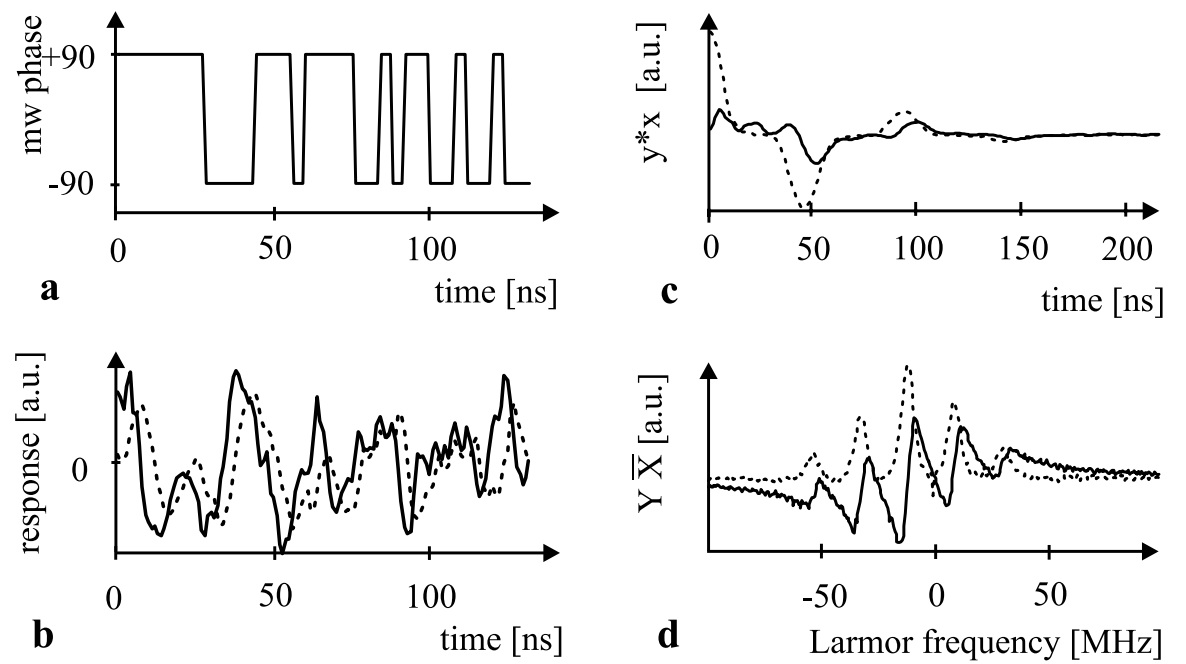

Fig. 9. Fourier-transform EPR at $3.4 \mathrm{~T} / 95 \mathrm{GHz}$ on the nitronyle-nitroxide radical [27] using broad-band stochastic excitation. (a): First $200 \mathrm{~ns}$ of a $1 \mu \mathrm{s}$ long pseudostochastic excitation sequence $x(t)$. The phase of the microwave is modulated according to this sequence. The FWHM bandwidth is $250 \mathrm{MHz}(\mathbf{b})$ : Response $y(t)$ of the spin system (solid: in phase, dashed: out-of-phase component). (c): cross-correlation of $x$ and $y, x * y$. The first 5 ns have been reconstructed using linear prediction. (d): Fourier transform of $x * y$. The dashed line is equivalent to the absorption spectrum of the two coupled nitrogens, the solid line is equivalent to the dispersion spectrum

tral bandwidth of this sequence is $250 \mathrm{MHz}$, similar to the bandwidth of a $4 \mathrm{~ns}$ pulse. While by using a single pulse the whole excitation energy has to be concentrated at once, by using the pseudostochastic excitation the excitation energy is spread over time. Thereby the required peak power is reduced as discussed by Ernst and Kaiser (approximately, the time average of the power must be the same). As an example of stochastic EPR, the response of a nitronyle-nitroxide molecule to the applied pseudostochastic sequence is shown in Fig. 9b. It was digitized, and the cross-correlation with the excitation, shown in Fig. 9c, was performed by computer. The cross -correlation is equivalent to the FID, and its Fourier transformation, shown in Fig. 9d, is equivalent to the EPR spectrum. Using quadrature detection, the spectra are obtained in absorption as well as in dispersion (dotted and solid lines).

Although a highly esthetic and intellectually stimulating approach, the experimental realization of stochastic EPR is difficult. In contrast to standard pulsed EPR, many problems are created by the simultaneous excitation and detection. Therefore, it is necessary to decouple excitation and detection modes using bimodal resonators. Furthermore, for samples with relaxation times shorter than $100 \mu \mathrm{s}$, the currently available microwave power of $5 \mathrm{dBm}$ is still below optimum. However, so far it could be shown, that although technically difficult due to the high time resolution required, the experimental bandwidth corresponds 
to that of the excitation and that the sensitivity is equal to the theoretically expected sensitivity. Based on the obtained results it could be estimated under which conditions the particularly interesting two dimensional (2D) high-field Fourier-transform EPR experiments are feasible [27]. These types of measurements, known as COSY type experiments in NMR, could for example reveal spin couplings in two-spin systems such as radical pairs. The stochastic approach to multidimensional NMR was discussed and realized by Blümich and Ziessow (see $[28,29])$.

\section{Conclusion}

Pulsed high-field EPR is used now in many areas of spectroscopy in biology, chemistry and physics. One rapidly growing field of application is the study of structure and dynamics of proteins and (bio)organic molecules. A small selection of it has been presented in this article. Even when EPR methods may be not so generally applicable and have to be designed for the respective project, the obtained information is extremely valuable and complementary to X-ray crystallography and NMR. Different from X-ray crystallography, with EPR one can probe the molecular complexes in their functional states and environments, and studies of their dynamic behavior are also possible. Furthermore, the environment of paramagnetic centers is probed in a more direct way and it is even possible to investigate cofactors of electron transfer systems 'in action'. Contrary to NMR, in EPR cw and pulsed methods will continue to coexist. In concluding it is fair to state that time-resolved and pulsed multifrequency EPR - in particular at high magnetic fields - has matured over recent years to present protein X-ray crystallographers and protein NMR spectroscopists with a powerful new ally for determining structure and dynamics of large biosystems.

\section{Acknowledgements}

It is a pleasure to acknowledge the cooperation of Thomas Prisner and Martin Rohrer (now at University Frankfurt) as well as of Harry Kurreck, Alexander Schnegg and Martin Fuchs from the FU Berlin during the projects touched in this article. Financial support by the Deutsche Forschungsgemeinschaft and Volkswagenstiftung is gratefully acknowledged.

\section{References}

1. N. M. Atherton: Principles of Electron Spin Resonance (Ellis Horwood, Chichester, 1993)

2. A. Carrington, A. D. McLachlan: Introduction to Magnetic Resonance (Harper \& Row, New York, Evanston, London, 1969)

3. R. R. Ernst, G. Bodenhausen, A. Wokaun: Principles of Nuclear Magnetic Resonance in One and Two Dimensions (Clarendon Press, Oxford, 1987) 
4. A. Schweiger, G. Jeschke: Principles of Pulse Electron Paramagnetic Resonance (Oxford University Press, Oxford, 2001)

5. T. F. Prisner, S. Un, R. G. Griffin: Isr. J. Chem. 32, 357 (1992)

6. T. F. Prisner: Adv. Magn. Opt. Reson. 20, 245 (1997)

7. 'Special Issue on High-Field EPR' . Appl. Magn. Reson. 16, pp. 106 - 308 (1999)

8. A. J. Hoff, J. Deisenhofer: Phys. Rep. 287, 1 (1997)

9. M. Rohrer, P. Gast, K. Möbius, T. Prisner: Chem. Phys. Lett. 259, 523 (1996)

10. A. G. Redfield: The Theory of Relaxation Processes (Academic Press, London, 1965) Vol. 1 of Adv. in Magn. Res. pp. 1-32

11. G. L. Millhauser, J. H. Freed: J. Chem. Phys. 81, 37 (1984)

12. S. A. Dzuba, Y. D. Tsvetkov, A. G. Maryasov: Chem. Phys. Lett. 188, 217 (1992)

13. S. A. Dzuba: Spectrochimica Acta A 56, 227 (2000)

14. S. Grimaldi, F. MacMillan, T. Ostermann, B. Ludwig, H. Michel, T. Prisner: Biochemistry 40, 1037 (2001)

15. A. Schnegg, M. Fuhs, M. Rohrer, W. Lubitz, T. Prisner, K. Möbius: 'Molecular Dynamics of $\mathrm{Q}_{A}^{-}$and $\mathrm{Q}_{B}^{-}$in Photosynthetic Bacterial Reaction Centers Studied by Pulsed W-band High-field EPR', in preparation

16. M. Rohrer, F. MacMillan, T. F. Prisner, A. T. Gardiner, K. Möbius, W. Lubitz: J. Phys. Chem. B 102, 4648 (1998)

17. M. Fuhs, G. Elger, A. Osintsev, A. Popov, H. Kurreck, K. Möbius: Molec. Phys. 98, 1025 (2000)

18. K. McLaughlan: 'Time-Resolved EPR' . In Advanced EPR, Applications in Biology and Biochemistry, ed. by A. J. Hoff (Elsevier, Amsterdam, 1989) pp. 345-369

19. D. Stehlik, K. Möbius: Annu. Rev. Phys. Chem. 48, 745 (1997)

20. P. J. Hore: 'Analysis of Polarized Electron Paramagnetic Resonance Spectra' . In Advanced EPR, Applications in Biology and Biochemistry, ed. by A. J. Hoff (Elsevier, Amsterdam, 1989) pp. 405-440

21. H. Kurreck, M. Huber: Angew. Chem. Int. Ed. Engl. 34, 949 (1995)

22. A. Berg, Z. Shuali, M. Asano-Someda, H. Levanon, M. Fuhs, K. Möbius, R. Wang, C. Brown, J. L. Sessler: J. Am. Chem. Soc. 121, 7433 (1999)

23. P. Piotrowiak: Chem. Soc. Rev. 28, 143 (1999)

24. T. F. Prisner, A. van der Est, R. Bittl, W. Lubitz, D. Stehlik, K. Möbius: Chem. Phys. 194, 361 (1995)

25. R. R. Ernst: J. Magn. Reson. 3, 10 (1970)

26. R. Kaiser: J. Magn. Reson. 3, 28 (1970)

27. M. Fuhs, T. Prisner, K. Möbius: J. Magn. Reson. 149, 67 (2001)

28. B. Blümich, D. Ziessow: J. Chem. Phys. 78, 1059 (1983)

29. B. Blümich: Prog. NMR Spectrosc. 19, 331 (1987) 